\title{
Accuracy of a Priority Medical Dispatch System in Dispatching Cardiac Emergencies in a Suburban Community
}

\author{
Michael J. Reilly, MPH, NREMT-P
}

Columbia University, Mailman School of Public Health, National Center for Disaster Preparedness, Center for Public Health Preparedness

\author{
Correspondence: \\ Michael J. Reilly, MPH, NREMT-P \\ Columbia University \\ 722 W. 186th Street, 10th Floor \\ New York, NY 10032 USA \\ E-mail: mr2381@columbia.edu
}

Keywords: advanced life support (ALS); emergency medical services (EMS); EMS communications systems; paramedics
Abbreviations:
ALS $=$ advanced life support
BLS = basic life support
$\mathrm{CMR}=$ computerized medical records
CVA $=$ cerebrovascular accident
$\mathrm{ED}=$ emergency department
$\mathrm{EMD}=$ Emergency Medical Dispatch
$\mathrm{EMS}=$ emergency medical services
$\mathrm{MPD}=$ Medical Priority Dispatch

Received: 04 February 2005

Accepted: 04 March 2005

Revised: 08 July 2005

Web publication: 23 March 2006

\begin{abstract}
Introduction: Over-triage of patients by emergency medical services (EMS) dispatch is thought to be an acceptable alternative to under-triage, which may delay how quickly life-saving care reaches a patient. Previous studies have looked at advanced life support (ALS) misutilization in urban- and countybased EMS systems and have attempted to analyze how dispatch methods either contribute to or alleviate this problem. ${ }^{1-5}$

Objective: The purpose of this study is to assess the relationship between dispatches of a cardiac nature in a Medical Priority Dispatch (MPD) system, and the actual clinical diagnosis as determined by an emergency department physician.

Methods: Calls for emergency medical assistance in a suburban community outside of a major metropolitan area were surveyed over a three-month period. Medical Priority Dispatch protocols determined that 104 of these calls were cardiac-related. Of these emergency calls, $56(53.8 \%)$ patients were transported to the local community hospital and treated by the emergency physician. A retrospective review of the medical records was conducted to determine whether the patient had a cardiac-related discharge diagnosis from the emergency department.

Results: Sixteen (28.6\%) of the patients in this cohort were diagnosed with a cardiac-related condition upon discharge from the emergency department. Forty $(71.4 \%)$ were diagnosed with a non-cardiac-related condition. The positive, predictive value of the dispatch protocol for the detection of an actual cardiac emergency in this EMS system was $28.6 \%$.

Conclusion: In this suburban community, the MPD system may over-triage emergency medical responses to cardiac emergencies. This can result in the only ALS (paramedic) unit in the community being unavailable in certain situations. Future studies should be conducted to determine what level (in any) of over-triage is appropriate in EMS systems using a MPD system.
\end{abstract}

Reilly MJ: Accuracy of a priority medical dispatch system in dispatching cardiac emergencies in a suburban community. Prebosp Disast Med 2006;21(1):77-81.

\section{Introduction}

Over the past 30 years, prehospital medical care has developed significantly into an efficient method for delivering out-of-hospital emergency medical services to millions of patients each year. The development of emergency medical services (EMS) regionalization, the creation and enhancement of trauma systems, and integrating EMS into the fire service have made advanced life support (ALS) [paramedic] services available in virtually every urban and suburban community in the United States.

The introduction of the enhanced 9-1-1 emergency system, and the development of the Emergency Medical Dispatch (EMD) and Medical Priority Dispatch (MPD) Systems, have been shown to improve EMS utilization, increase efficiency, and decrease the percentage of inappropriate ALS responses. ${ }^{1}$ However, there are operational flaws in these systems that contribute to and compound the problem of ALS misutilization. A study by Palumbo et al describes a "substantial disagreement" between dispatcher-assigned priorities of care and those of emergency physicians. ${ }^{2}$ Additionally, they assert 
that the MPD system has a tendency to over-triage EMS responses to a higher acuity, requiring a more frequent $A L S$ response.

Over-triage of patients by EMS dispatch is thought to be an acceptable alternative to under-triage, which may cause an undesirable patient outcome. With this in mind, the implications of over-triage on the availability of ALS resources must be acknowledged. The impact of over-triage will affect EMS systems differently depending on their design (ALS transporting, two-tiered, ALS non-transport, system status management, etc.). The over-utilization of ALS resources in a two-tiered EMS system leads to paramedic units remaining unavailable for extended periods of time. In communities with a limited availability of ALS units may result in a delay in accessing paramedic-level care, or having no paramedic units available for emergency response. If over-triage is thought to be an acceptable consequence of conservative EMD protocols, it is important for EMS systems to have operational plans in place to ensure that ALS care is available to the community when ALS units are out of service.

Previous studies have looked at the problem of ALS misutilization in urban- and county-based EMS systems and have attempted to analyze how dispatch methods either contribute to or alleviate this issue. ${ }^{1-5}$ This study was performed to investigate the problem of ALS misutilization in a suburban, northeastern US community that uses a MPD system. This investigation specifically assesses the relationship between the triage of a caller's emergent medical complaint by a civilian emergency medical dispatcher, and the patient's actual clinical diagnosis after being evaluated and treated by an emergency department physician.

\section{Methods}

Study Design

This was a retrospective, cross-sectional study of a suburban community outside a major metropolitan area. All calls to 9-1-1 for medical aid were surveyed over a three-month period (01 November 2001-31 January 2002). This study was approved by the Columbia University Medical Center Institutional Review Board: IRB \#AAAB3958.

\section{Population and Setting}

The city examined in this study is a suburban city outside of a major metropolitan area. The city covers an area of approximately 13 square miles and had a population of approximately 60,000 in the year 2000 . Emergency medical services are delivered to this community by a private EMS agency that provides the city with one basic life support (BLS) ambulance staffed with two certified emergency medical technicians, and one ALS ambulance staffed with two certified paramedics. The units are dedicated to the city 24-hours/day, and are dispatched to emergencies directly by the city's fire department dispatcher.

Emergency calls for medical aid are received by a municipal 9-1-1 call center staffed with trained civilian dispatchers, who take calls and dispatch medical responses by utilizing a certified emergency medical dispatch (EMD) protocol consisting of a MPD system.

The city has an EMS workgroup with members from the police department, fire department, EMS agency, and emergency call center. This workgroup is responsible for the EMS system's quality improvement and receives medical oversight from the local hospital's emergency department medical director. This workgroup is responsible for determining which units respond to which codes as entered into a computer by the emergency dispatchers. All modifications to this EMD system are endorsed and approved by the physician medical director prior to implementation. This study also involved the city's local community hospital. This 204-bed, acute-care hospital has a 24-hour emergency department and in-house specialties including cardiology, pulmonary medicine, orthopedics, pediatrics, psychiatry, and obstetrics-gynecology. This hospital did not have a cardiac catheterization unit during the study period. In this EMS system, the usual practice was for all patients with severe clinical presentations who were determined unable to tolerate extended transport to a specialty care facility, to be transported to this hospital for initial stabilization.

\section{Study Methods}

All calls to 9-1-1 for medical aid in the community were examined over a three-month period (01 November 2001-31 January 2002). Calls in which the dispatcher determined that the patient was experiencing: (1) chest pain; (2) heart problems; or (3) a cardiac/respiratory emergency were included in the study. Such calls specifically required an ALS response in the city's MPD system. The advantage to using these three combinations of dispatch codes is that the majority of calls with cardiac-related complaints would be triaged using the EMD protocol into these categories.

A second inclusion criterion was that only calls in which the patients were taken to the local community hospital were included in the study. Calls in which patients were transported to other hospitals were excluded from the study. Calls in which the patient refused prehospital treatment and transportation also were excluded. Finally, calls in which patient records could not be located or names were incorrectly entered into the computerized medical records (CMR) system were excluded.

A retrospective chart review was performed on the discharge diagnoses made by the emergency department physicians. These discharge diagnoses were compared to the response codes issued by the dispatch center. Since this was a retrospective study, the emergency physicians did not know at the time of patient care that these cases would be investigated, and, therefore, could not influence the study outcomes.

Calls received by the emergency dispatch center were cross-referenced by call number, response times, and addresses with the EMS patient care reports to establish the patient's initial clinical presentation and the transport hospital destination. Patients transported to the local hospital with the dispatch codes described earlier were sorted and cross-referenced with the hospital's computerized medical records (CMR) system to identify the emergency department discharge diagnosis and the patient's disposition.

Emergency department discharge diagnoses that were considered to be cardiac-related included: (1) chest pain-rule-out myocardial infarction; (2) unstable angina; (3) myocardial infarction; and (4) cardiac miscellaneous (e.g., congestive heart failure, and dysrhythmia). Non-cardiac related emergency department discharge diagnoses included: (1) non-cardiac 


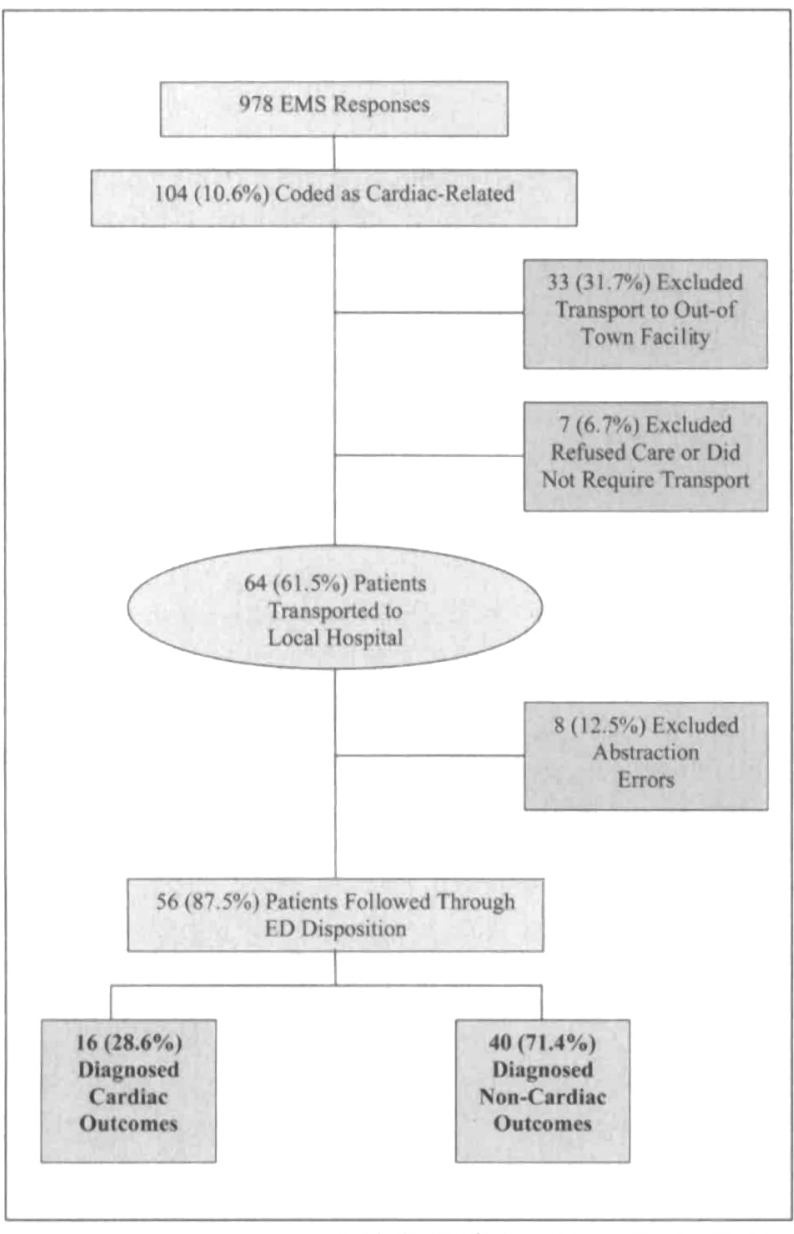

Reilly 두 2006 Prehospital and Disaster Medicine

Figure 1-Flow diagram of participants in study $(\mathrm{ED}=$ emergency department; EMS = emergency medical services)

chest pain (e.g., pleuritic or musculo-skeletal discomfort); (2) abdominal pain; (3) gastrointestinal illness; (4) intoxication; (5) anxiety; (6) seizure; (7) miscarriage; (8) hyperglycemia; (9) syncope; (10) dizziness; and (11) cerebrovascular accident.

All data were compiled and analyzed in an Excel 2000 (Microsoft, Inc., Redmond, Washington) spreadsheet using basic descriptive statistical methods. Positive predictive value of the dispatch protocol was calculated in the standard manner.

\section{Results}

During the three-month study period, 978 calls for medical assistance were received. A total of 104 calls were coded as either: (1) chest pain; (2) heart problems; or (3) a cardiac/respiratory emergency. A total of 64 (61.5\%) patients were transported to the local community hospital. The remaining 33 patients were taken to other hospitals or academic medical centers based on a variety of factors such as patient request, hospital diversion status, EMS provider discretion, and/or requirements for specialized care (i.e., cardiac catheterization). Additionally, seven (6.7\%) patients were omitted from the study because the patient refused prehospital treatment and transportation.
After limiting the sample to only those patients transported to the local hospital, eight (12.5\%) additional patients were excluded because their patient records could not be located, or their names were entered incorrectly into the CMR system, making it impossible to retrieve outcome-related data. Patient distribution by classification used in this study is in Figure 1 .

It is important to point out that in this EMS system, patients showing severe clinical presentations who were determined by prehospital providers to be unable to sustain the extended transport to a tertiary care facility would be transported to the local community hospital for critical care intervention and stabilization. Due to the large percentage of patients who were transported to the local community hospital, and because this hospital received a heterogeneous mix of clinical severity, the patients who were transported to other facilities were excluded from this study. This decision also assisted in the collection process of obtaining outcome data. The number of patients that were excluded based upon receiving facility totaled 33, leaving 71 patients potentially eligible for the study.

The prevalence of discharge by diagnosis from the 56 calls included in the study. Emergency department physicians diagnosed that $16(28.6 \%)$ of the patients had cardiacrelated conditions. In $40(71.4 \%)$ of these calls, the patients were diagnosed with non-cardiac-related ailments. As a tool for the screening of callers with potentially cardiac-related ailments, the caller triage and dispatch protocol has a positive predictive value of $28.6 \%(16 / 56)$.

\section{Discussion}

A total of $40(71.4 \%)$ of the calls included in this study turned out not to be cardiac in origin as the MPD system initially had classified. Several studies have suggested that emergency responses for medical aid that are dispatched using a MPD system or EMD protocol may not accurately reflect the nature of the illness or injury. $1,4-6$

This study suggests that use of the EMD protocol used in this EMS system results in over-triage of ALS responses to potential cardiac-related emergencies. Use of this process may miss the nature of medical emergencies to the responding public safety units. It is understood that not all chest pain calls are cardiac-related, and not all patients who are triaged by an emergency medical dispatcher into the cardiac/respiratory arrest/death category have cardiacrelated problems. Nevertheless, this study underscores a public safety and public health problem that exists not only in the urban and rural communities cited in other studies, but in suburban communities as well. ${ }^{1-6}$

The inappropriate utilization of EMS and the unnecessary depletion of available ALS resources for non-emergency patients can tax a scarce community resource. This can be a source of frustration for providers, medical directors, emergency department physicians, and system administrators. Many believe that over-triage is acceptable, if it is likely to ensure that true ALS emergencies are not missed by undertriaging an emergency response. If this is true, it is important to assess how much over-triage is acceptable, and if patient care is affected by allocating resources in this manner. 


\begin{tabular}{|c|c|}
\hline ED discharge diagnosis & $\begin{array}{c}\text { Number }(\%) \\
n=56\end{array}$ \\
\hline Chest Pain R/O Ml & $11(20)$ \\
\hline Unstable Angina & $1(2)$ \\
\hline Myocardial Infarction (MI) & $2(4)$ \\
\hline Cardiac Miscellaneous & $2(4)$ \\
\hline Non-Cardiac Chest Pain & $7(13)$ \\
\hline Respiratory & $10(18)$ \\
\hline Abdominal Pain & $4(7)$ \\
\hline GI Illness & $2(4)$ \\
\hline Alcohol Intoxication & $6(11)$ \\
\hline Anxiety & $4(7)$ \\
\hline Seizure & $1(2)$ \\
\hline Miscarriage & $1(2)$ \\
\hline Hyperglycemia & $1(2)$ \\
\hline Toxic Effects & $1(2)$ \\
\hline Syncope & 1 (2) \\
\hline Dizziness & $1(2)$ \\
\hline CVA & 1 (2) \\
\hline
\end{tabular}

Table 1-Emergency department discharge diagnoses among study participants $(\mathrm{CVA}=$ cerebrovascular accident; $\mathrm{ED}$ = emergency department; $\mathrm{GI}=$ gastrointestional $\mathrm{R} / \mathrm{O}=$ rule-out)

Dispatch strategies have been examined and proposed by public safety professionals with the goal of reducing unnecessary EMS responses and reducing the misutilization of ALS. ${ }^{1,3,5}$ One factor that is difficult to control in a MPD system is the ability of dispatchers to decide subjectively how to allocate the necessary resources to a caller's emergency. Because of variations in patients' symptoms, the ability of a caller to communicate the necessary information, and the dispatcher's personal interpretation of the nature or severity of a callers' complaint, the sensitivity and specificity of the MPD system is called into question.

Possible solutions may include: (1) improving caller screening; (2) utilizing an all-ALS response system; (3) sending a BLS unit on all calls and developing a triage protocol for ALS providers to ensure that paramedics remain available if not needed; (4) not requiring an ALS unit to transport a non-emergent patient when a.BLS unit is available within a reasonable response distance and time; (5) developing a system for patients to access more acute but non-emergency medical care in situations in which they have no means of transportation or primary medical provider to contact; and (6) ensuring visiting nurses are available for "urgent-not-emergent", in-home consultations when primary care is not available and/or the patient is unable to travel outside the home to visit a physician.

\section{Limitations}

In this analysis, some situations may have introduced bias and/or confounding factors. The sampling frame consisted of 978 patients, of which 104 were selected as the sample of interest. This number of patients was reduced to 56 in the final cohort. The study's rigor could have been increased by studying multiple, suburban communities within a county or EMS region in the metropolitan area under study. This study assessed one-quarter of a calendar year. If this study is replicated, it may be beneficial to examine a larger group of patients or to gather data over a longer period of time.

Selection bias also may have been introduced by only including those patients who were transported to the local community hospital. For example, patients with a significant cardiac history may have requested a specific specialty hospital, or the paramedics may have chosen a transport destination with the capability to perform interventional cardiology. Selection bias could have been limited by including every transport destination, or by assigning an alternative sampling method that would yield a representative number of patients that went to each of the different receiving facilities.

Clerical and technical error played a role in this study through the exclusion of eight patients due to the inability to locate their medical records. Without knowing their diagnostic outcomes, it is impossible to determine their impact on the study results. Misclassification error may have occurred if the dispatcher entered the wrong response code into the computer, resulting in an inappropriate response. Such an error actually would be a contributing factor to the problem under study in this investigation. Additionally, because the MPD protocols that were selected for this study are not designed exclusively for cardiac symptoms, it is possible that non-cardiac patients may have been triaged into this study cohort. Future studies that attempt to assess the validity and/or overall utility of a MPD system should use more rigorous study methodology to ensure that the system is depicted accurately in relation to its intended operational capabilities.

Future studies should assess whether unavailable ALS units would contribute to increased morbidity and mortality. Additionally, studies that look at the compliance with an EMD protocol by a dispatch center should be conducted. Furthermore, any proposed plan or model to deliver non-emergency, out-of-hospital medical care would be useful in attempting to resolve issues of ALS misutilization.

\section{Conclusion}

In this suburban community, the medical priority dispatch system over-triaged ALS calls for a potential cardiac emergency $>70 \%$ of the time. In a community with only one dedicated ALS unit, over-triage could prevent ALS care from reaching patients who could benefit from ALS interventions. Assuming that to an extent, over-triage is acceptable in order to prevent missed ALS calls, future studies should determine what level of over-triage is acceptable, how EMS and MPD systems are utilized in suburban EMS systems, and how often ALS resources are unable to respond to emergencies when sent on an inappropriate response.

\section{Acknowledgements}

The author thanks Dr. David Cone, MD, Dr. David Markenson, MD EMT-P, and David Becker, MPA/H, NHA, EMT-B for their assistance in the preparation of this manuscript. 
References

1. Bailey ED, O'Connor RE, Ross RW: The use of emergency medical dispatch protocols to reduce the number of inappropriate scene responses made by advanced life support personnel. Prehosp Emerg Care 2000;4:186-189.

2. Palumbo L, Kubincanek J, Emerman C, et al: Performance of a system to determine EMS dispatch priorities. Am J Emerg Med 1996;(14)4:388-390.

3. Dale J, Higgins J, Williams $\mathrm{S}$, et al: Computer assisted assessment and advice for "non-serious" 999 ambulance service callers: The potential impact on ambulance dispatch. Emerg Med J 2003;20:178-183.
4. Neely KW, Eldurkar JA, Drake MER: Do emergency medical services dispatch nature and severity codes agree with paramedic field findings? Acad Emerg Med 2000;7:174-180.

5. Neely KW, Norton RL, Schmidt TA: The strength of specific EMS dispatcher questions for identifying patients with important clinical field findings. Prebosp Emerg Care 2000;4:322-326.

6. Richards JR, Ferrall SJ: Inappropriate use of emergency medical services transport: Comparison of provider and patient perspectives. Acad Emerg Med 1999;6:14-20. 